\section{Fire-Eater's Pneumonia: a likely hazard not uncommon in fire eaters}

\section{Dear Editor,}

Even if chemical pneumonia due to aspiration of hydrocarbon fuel is considered an uncommon hazard, their real incidence is unknown since only patients with major symptoms go to the doctor. The literature is mostly composed of some case reports $[1,2]$. Radiologic findings are evocative but aspecific and their interpretation is supported by recent exposure to hydrocarbons. Computed tomography has a basic role in early identification of life-threatening features. Prompt cure is strongly suggested by the possible evolution to serious life threatening pleuropulmonary complications assessed on CT follow-up. Risk unawareness among fire eaters may delay both clinical diagnosis and treatment.

A 25-year-old male unknowingly aspirated a small amount of liquid hydrocarbon fuel while performing first attempt at fire-breathing. In the next days he developed shortness of breath, dry cough and fever. He went to the hospital only seven days after the performance. A plain chest radiograph showed retrocardiac and middle lobe airspace consolidations and a provisional diagnosis of community acquired pneumonia was performed in emergency medicine. Exogenous lipoid pneumonia was diagnosed after referral to an internal medicine unit, where the characteristic clinical history was finally disclosed by the patient. On admission, auscultation of the lungs revealed decreased breath sounds and coarse crackles over the bases. Remaining physical examination was unremarkable. Laboratory data showed increased in- flammatory parameters. Arterial blood gases measurements were within normal ranges. Computed tomography showed extensive bilateral pulmonary consolidations and peripheral ground glass opacities in the left lower lobe with mediastinal hilar lymphadenopathy (fig 1a). The patient was given antibiotics (amoxicillin-clavulanate, clarithromicin) and steroids. The diagnostic work-up included microbiological and lung function tests, optic fibre bronchoscopy and an analysis of bronchoalveolar lavage fluid. The blood culture results were negative. The bronchoalveolar lavage specimen showed neutrophilia and vacuolated macrophages oil red positive. The treatment was not discontinued until a clinical improvement was obtained. The patient showed a gradual and regular clinical course and he was dicharged symptom-free 10 days after the admission. A follow-up chest CT four weeks later merely showed rare streaky-fibrotic residues (fig $1 \mathrm{~b}$ ).

Exogenous lipoid pneumonia may be either chronic [3] or acute [4]. Acute exogenous lipoid pneumonia is better known as fire-eater' pneumonia. Even if fire-eaters' pneumonia is considered a relatively uncommon condition following hydrocarbon aspiration, we really do not know the incidence of the event, since people may be unware of inhaling any part of it and does not always adequately consider the risk of devoloping pneumonia with associated complications [5]. Only major symptoms including dyspnea, cough, chest pain, fever, shortness of the breath bring to the doctor. History is the key to recognising otherwise aspecific radiologic findings. They usually disappear after treatment with antibiotics and steroids and persistent lung injury is unusual. Some cases spontaneously resolve themselves without any active treatment after the offending agent has been ceased. Lung function generally returns to normal within 2-3 weeks. Mortality is less than 1\%. Some-

Fig. 1a. - Axial CT (lung window level) showing a small consolidation in the middle lobe and a focal area of ground glass attenuation in the left lower lobe (arrow heads). b. - Axial CT (lung window level) showing the almost completely disappearance of both pulmonary consolidations and thickened parenchimal area.

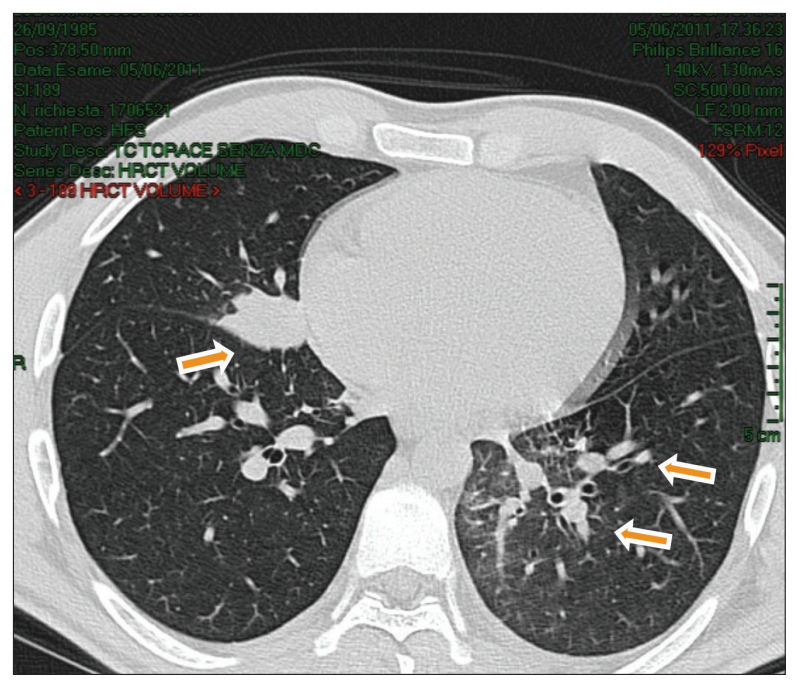

Fig. 1a. - Middle lobe consolidation and left lower lobe ground glass attenuation (arrow heads).

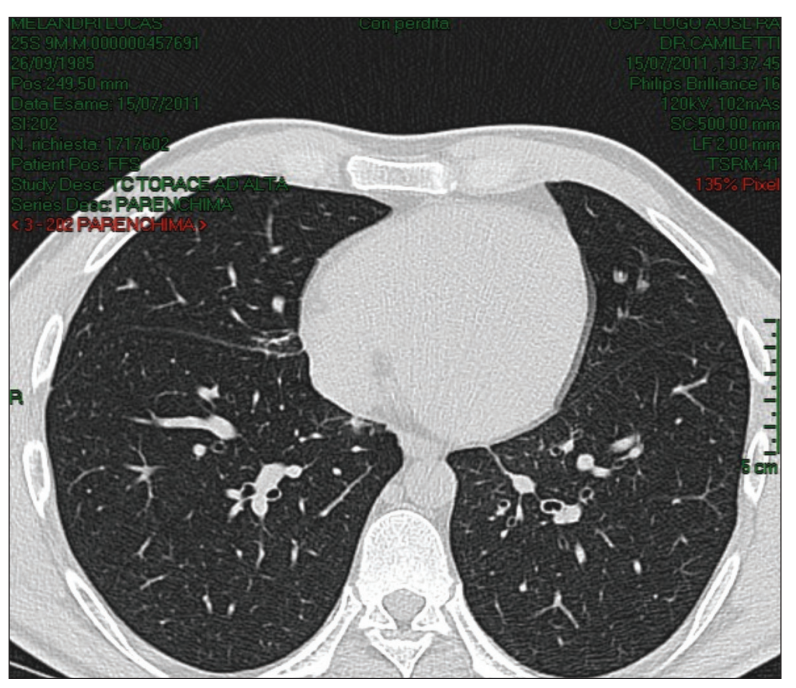

Figure 1b. - Disappearance of both pulmonary consolidations and left lower lobe thickened parenchimal area. 
times, fire-eaters' pneumonia results in severe pleuropulmonary complications, such as necrotising consolidations, widespread cavitations, large pleural effusions, bronchopleural fistulae and spontaneous pneumothorax.

In our case, the diagnostic confirmation was delayed by the initial low level of consciousness of his discare of the patient. In any event, regular assessment and follow-up with CT was mandatory, since, ever unusual, pleuropulmonary complications in patients with fire eaters' pneumonia are possible and should be detected early and adequately managed. This is a good reason for immediate administration of antibiotics associated with an adequate supportive care.

Acknowledgements: We are indebted to dr. R. Montanari \& dr. M. Puccetti for their invaluable support in this study: RM evaluated the chest radiograph and computed tomography scan. MP examined the bronchoalveolar lavage fluid specimen.

\section{G. Re, P. Candoli, F. Dazzani}

Department of Internal Medicine, Lugo Hospital, Italy. Correspondence: Dr. Giuseppe Re, Viale Dante 10, 48022 Lugo (RA), Italy; e-mail: g.re@ausl.ra.it

\section{References}

1. Grossi E, Crisanti E, Poletti G, Poletti V. Fire-eater's Pneumonitis. Monaldi Arch Chest Dis 2006; 65: 59-61.

2. Guandalini M, Steinke K. Fire eating: hazards of hydrocarbon aspiration. Australasian Radiology 2007; 51: 567-569.

3. Simmons A, Rouf E, Whittle J. Not your typical pneumonia: a case of exogenous lipoid pneumonia. J Gen Intern Med 2007; 22: 1613-6.

4. Dell'Omo M, Murgia N, Chiodi M, et al. Acute pneumonia in fire eater. Int J Immunopathol Pharmacol 2010; 23: 1289-1292.

5. Yigit O, Bektas F, Vefa Sayrac A, Senay E. Fire-eater's pneumonia: two case reports of accidentally aspirated paraffin oil. J Emerg Med 2012; 42: 417-419.

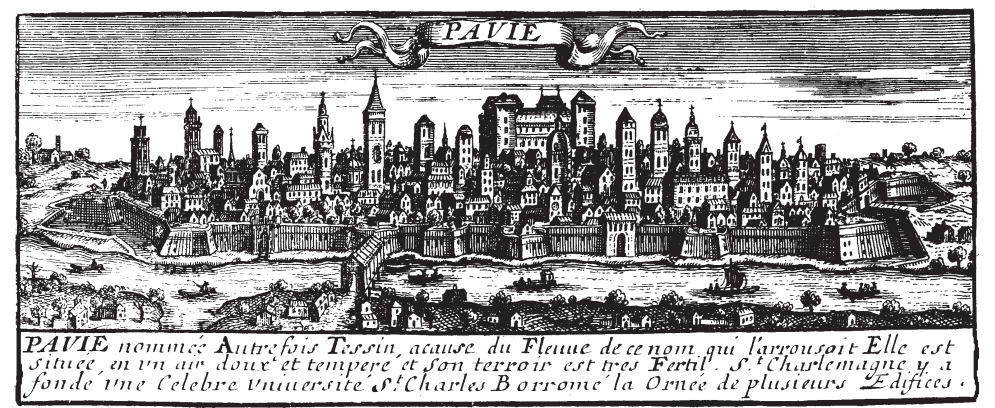

